\title{
Chronic thyroiditis in patients with advanced breast carcinoma: metabolic and morphologic changes on PET-CT
}

\author{
Ukihide Tateishi $\cdot$ Cristina Gamez • \\ Shaheenah Dawood • Henry W. D. Yeung • \\ Massimo Cristofanilli • Tomio Inoue • \\ Homer A. Macapinlac
}

Received: 11 September 2008 / Accepted: 5 December 2008

(C) Springer-Verlag 2009

\begin{abstract}
Purpose To investigate clinical implications of FDG uptake in the thyroid glands in patients with advanced breast carcinoma by comparing metabolic and morphologic patterns on positron emission tomography (PET)/computed tomography $(\mathrm{CT})$.

Methods The institutional review board waived the requirement for informed consent. A retrospective analysis was performed in 146 women (mean age 54 years) with advanced breast carcinoma who received systemic treatment. All patients underwent PET-CT before and after treatment. All PET-CT studies were reviewed in consensus by two reviewers. Morphologic changes including volume and mean parenchymal density of the thyroid glands were evaluated. Maximum standardized uptake value (SUVmax) and total
\end{abstract}

\footnotetext{
U. Tateishi $\cdot$ C. Gamez $\cdot$ H. W. D. Yeung $\cdot$ H. A. Macapinlac Department of Nuclear Medicine, University of Texas, MD Anderson Cancer Center,

Houston, TX, USA

U. Tateishi $\cdot$ T. Inoue

Department of Radiology,

Yokohama City University Graduate School of Medicine,

Yokohama, Japan

S. Dawood $\cdot$ M. Cristofanilli

Division of Breast Medical Oncology, University of Texas,

MD Anderson Cancer Center,

Houston, TX, USA

U. Tateishi $(\square)$

Division of Diagnostic Imaging,

University of Texas MD Anderson Cancer Center,

Unit 1263, 1515 Holcombe Blvd.,

Houston, TX, USA

e-mail: utateish@yokohama-cu.ac.jp
}

lesion glycolysis (TLG) were determined to evaluate metabolic changes. These parameters were compared between patients with chronic thyroiditis who received thyroid hormone replacement therapy and those who did not. Results Of the 146 patients, 29 (20\%) showed bilaterally diffuse uptake in the thyroid glands on the baseline PET-CT scan. The SUVmax showed a linear relationship with volume $(r=0.428, p=0.021)$ and the mean parenchymal density $(r=-0.385, p=0.039)$ of the thyroid glands. In 21 of the 29 patients $(72 \%)$ with hypothyroidism who received thyroid hormone replacement therapy, the volume, mean parenchymal density, SUVmax, and TLG of the thyroid glands showed no significant changes. In contrast, 8 of the 29 patients $(28 \%)$ who did not receive thyroid hormone replacement therapy showed marked decreases in SUVmax and TLG.

Conclusion Diffuse thyroid uptake on PET-CT represents active inflammation caused by chronic thyroiditis in patients with advanced breast carcinoma. Diffuse thyroid uptake may also address the concern about subclinical hypothyroidism which develops into overt disease during follow-up.

Keywords FDG $\cdot$ PET P PET/CT P PET oncology · Clinical trials

\section{Introduction}

Fluorine-18-fluorodeoxyglucose (FDG) positron emission tomography/computed tomography (PET-CT) is a noninvasive whole-body imaging technique used to evaluate many kinds of malignant tumors. The results of previous studies suggest that incidental uptake in the thyroid glands is often encountered in daily practice [1]. Some patterns of thyroid 